\title{
Rechtsprechungswegweiser Europarecht 2012:
}

\author{
Aktuelle EuGH-Rechtsprechung mit Prüfungsrelevanz \\ Von Patricia Sarah Stöbener, LL.M., Angela Regina Stöbener, LL.M., und Dr. Mattias Wendel, Berlin*
}

Der Beitrag dient der Prüfungsvorbereitung im Fach Europarecht, indem er einen didaktisch aufbereiteten Überblick über die aus Ausbildungsperspektive besonders relevanten Rechtsprechungsentwicklungen der letzten zweieinhalb Jahre schafft.

\section{Einleitung}

Die Prüfungsvorbereitung im Fach Europarecht gilt Studenten und Referendaren häufig als schwieriges Unterfangen. Grund dafür ist nicht so sehr die Anforderung, sich bei knapp bemessener Zeit das ausbildungsrelevante Grundwissen im Europarecht anzueignen. Hier kann auf eine Vielzahl an Lehr- und Fallbüchern zurückgegriffen werden. Probleme bereitet vielmehr der Umstand, sich über das Lehrbuchwissen hinaus hinreichend mit den jüngsten Rechtsprechungsentwicklungen auseinanderzusetzen. Kenntnisse aktueller EuGH-Entscheidungen und jüngster Rechtsprechungsentwicklungen sind im Fach Europarecht indes unerlässlicher Bestandteil einer gelungenen Prüfungsvorbereitung, sei es für die Schwerpunktklausur oder für die mündliche Prüfung im Ersten oder Zweiten Staatsexamen.
Zu einer solchen Prüfungsvorbereitung möchte der folgende Beitrag eine Hilfestellung geben. Die ausgewählten Urteile, die im Wesentlichen aus dem Zeitraum Anfang 2010 bis Ende 2011 stammen, werden komprimiert und unter Berücksichtigung der üblichen Prüfungsreihenfolge dargestellt. Indem auch die zentralen Vorgängerentscheidungen im Text oder in den Fußnoten Erwähnung finden, können gleichzeitig die zentralen Leitentscheidungen des EuGH in Erinnerung gerufen werden.

Mit Blick auf europarechtliche Prüfungen besonders interessant ist die Rechtsprechungsentwicklung zur Unionsbürgerschaft und zum allgemeinen Freizügigkeitsrecht. Prüfungsklas-

\footnotetext{
* Patricia Sarah Stöbener, LL.M. (King's College London) ist Kollegiatin im Graduiertenkolleg »Verfassung jenseits des Staates« und Research Fellow am Walter Hallstein-Institut für Europäisches Verfassungsrecht an der HumboldtUniversität zu Berlin. Angela Regina Stöbener, LL.M. (King's College London) ist Assessorin und promoviert an der Freien Universität Berlin, Lehrstuhl Prof. Dr. Christian Armbrüster. Dr. Mattias Wendel, Maîtr. en droit (Paris 1) ist wissenschaftlicher Assistent am Walter-Hallstein-Institut für Europäisches Verfassungsrecht der Humboldt-Universität zu Berlin, Lehrstuhl Prof. Dr. Dr. h.c. Ingolf Pernice. Teil 2 und 3 des Beitrags erscheinen in den nächsten beiden JURA Heften. Stand des Beitrags ist der 19. 4. 2012.
} 
siker sind seit jeher die Grundfreiheiten, die vor diesem Hintergrund ebenfalls eingehend behandelt werden. Hier werden die allgemeinen Entwicklungen vorab dargestellt. Danach folgen Fälle zu den einzelnen Grundfreiheiten. Schließlich folgen noch einige Fälle zur Diskriminierung aus anderen Gründen als der Staatsangehörigkeit, zum Anwendungsbereich der Unionsgrundrechte, zum Beihilfenrecht und zu Fragen des Prozessrechts. Die abschließenden Fälle zum Verbraucherrecht können dem Prüfling sowohl im Europarecht als auch im Zivilrecht begegnen.

\section{Unionsbürgerschaft und allgemeines Freizügigkeitsrecht}

Die EuGH-Rechtsprechung zur Unionsbürgerschaft (Art.20 AEUV) und zum allgemeinen Freizügigkeitsrecht (Art. 21 AEUV) ist in den vergangenen Jahren durch eine besondere Entwicklungsdynamik gekennzeichnet. In seiner Prüfungsrelevanz ist dieser Themenkomplex mittlerweile dem der Grundfreiheiten ebenbürtig. Der EuGH hat in einer ganzen Reihe von Urteilen das an die Unionsbürgerschaft geknüpfte Freizügigkeitsrecht zusehends zu einer »Grundfreiheit ohne Markt « ${ }^{1}$ fortentwickelt. Dies umfasst allem voran die prätorische Entfaltung von Art. 21 AEUV hin zu einem Beschränkungsverbot, welches insbesondere in Wegzugssituationen relevant wird. Eine Beschränkung der Freizügigkeit durch den Herkunftsmitgliedstaat liegt nach der mittlerweile gefestigten Rechtsprechung des Gerichtshofs dann vor, wenn die innerstaatliche Maßnahme geeignet ist, den Unionsbürger von der Ausübung seines Freizügigkeitsrechts tatsächlich oder potenziell abzuhalten ${ }^{2}$. Während in der klassischen Wegzugssituation der Unionsbürger, der sich gegenüber seinem Heimatstaat auf Art. 21 AEUV beruft, von seiner Freizügigkeit Gebrauch gemacht hat oder dies zumindest potenziell beabsichtigt, hatte sich der EuGH in zwei Urteilen im Jahre 2011 mit Fallgestaltungen auseinanderzusetzen, in denen es an einem solchen Grenzübertritt fehlte. Der Gerichtshof nahm dies zum Anlass, neue Akzente zu setzen. Im Übrigen entwickelte er seine Rechtsprechung im Bereich der Inländergleichbehandlung (Art. 18 i. V. m. Art. 21 AEUV), der sog. »Namensfälle s sowie zum aufenthaltsrechtlichen Sekundärrecht fort.

\section{Aufenthaltsrecht}

\section{a) Urteil Ruiz Zambrano}

Ein aufsehenerregendes Urteil erging zum Aufenthaltsrecht für drittstaatsangehörige Eltern minderjähriger Unionsbürger im Fall Ruiz Zambrano ${ }^{3}$. Herr Ruiz Zambrano und seine Ehefrau, beide kolumbianische Staatsangehörige, bekamen während ihres laufenden Asylverfahrens in Belgien zwei Kinder, welche die belgische Staatsangehörigkeit erhielten. In einem Verfahren über einen Anspruch Herrn Zambranos auf Arbeitslosengeld stellte sich die Frage, ob er ein Aufenthaltsrecht in Belgien hatte.

Im Rahmen der - systematisch vorrangigen - Prüfung von Sekundärrecht stellt der EuGH zunächst fest, dass die sog. Freizügigkeitsrichtlinie 2004/38 nicht auf den Fall anwendbar ist. Nach Art. 3 I gilt diese Richtlinie »für jeden Unionsbürger, der sich in einen anderen als den Mitgliedstaat, dessen Staatsangehörigkeit er besitzt, begibt oder sich dort aufhält, sowie für seine Familienangehörigen, die ihn begleiten oder ihm nachziehen«. Da die Kinder belgische Staatsangehörige waren, schied Belgien als »anderer « Mitgliedstaat aus. Außerdem hatten die Kinder von ihrem Recht auf Freizügigkeit im Gebiet der
Mitgliedstaaten niemals Gebrauch gemacht ${ }^{4}$. Es fehlte also an einem die Anwendbarkeit des Sekundärrechts eröffnenden, grenzüberschreitenden Bezug.

Indes bejaht der Gerichtshof ein derivatives - dh in diesem Fall vom Unionsbürgerstatus des Kindes abgeleitetes - Aufenthaltsrecht direkt aus der Unionsbürgerschaft nach Art. 20 AEUV (nicht Art. 21 AEUV!). Hierzu rekapituliert der EuGH seine ständige Rechtsprechung, wonach der Unionsbürgerstatus dazu bestimmt sei, der grundlegende Status der Angehörigen der Mitgliedstaaten zu sein ${ }^{5}$. Deshalb stehe Art. 20 AEUV nationalen Maßnahmen entgegen, die bewirken, dass den Unionsbürgern der tatsächliche Genuss des Kernbestands der Rechte, die ihnen der Unionsbürgerstatus verleiht, verwehrt wird. Eine derartige Situation liege jedenfalls in einer Konstellation wie der des Ausgangsverfahrens vor. Denn eine Aufenthaltsverweigerung für die drittstaatsangehörigen Eltern habe zur Folge, dass deren minderjährige Kinder - Unionsbürger faktisch gezwungen seien, das Gebiet der Union zusammen mit ihren Eltern zu verlassen. Dann aber wäre es, so der Gerichtshof, den genannten Unionsbürgern unmöglich, den Kernbestand der Rechte, die ihnen der Unionsbürgerstatus verleiht, tatsächlich in Anspruch zu nehmen ${ }^{6}$.

Die Besonderheit des Falles besteht also darin, dass der EuGH durch den Rekurs auf die Verletzung des sog. Kernbestandes der Unionsbürgerrechte der Kinder ein derivatives Aufenthaltsrecht ihrer drittstaatsangehörigen Eltern gewährt, obwohl es an einem grenzüberschreitenden Bezug fehlt. Im Gegensatz zu früheren Fällen, wie den sog. Namensfällen (s. u.) oder gewöhnlichen Wegzugsfällen, wird nicht auf die Freizügigkeit des Art. 21 AEUV, sondern auf Art. 20 AEUV abgestellt, weil es nicht um die (potentielle) Beschränkung der innereuropäischen Freizügigkeit des Kindes, sondern den (grundrechtlichen) Unionsbürgerstatus als solchen geht. Dies war zuvor nur einmal in Rottmann angedeutet worden, wo auf den Verlust des Status als Unionsbürger und die damit verbundenen Rechte abgestellt und eine Verhältnismäßigkeitsprüfung durchgeführt wurde, jedoch noch nicht vom » Kernbereich « die Rede war?.

\section{b) Urteil McCarthy}

Es war nur eine Frage der Zeit, dass der EuGH die Grenzen dieser Kernbereichsrechtsprechung würde ausloten müssen. Erste Gelegenheit hierzu bot der Fall McCarthy ${ }^{8}$. Frau McCarthy, eine Staatsangehörige des Vereinigten Königreichs, die von Geburt an dort gelebt hatte, besaß neben der britischen auch die irische Staatsangehörigkeit. Sie heiratete einen nicht aufenthaltsberechtigten jamaikanischen Staatsangehörigen. Sie und ihr Ehemann beantragten sodann eine Aufenthaltserlaubnis

1 Wollenschläger Grundfreiheit ohne Markt, 2007.

2 Vgl zB EuGH Urt v 18. 7. 2006, C-406/04, Slg 2006, I-6947, Rdn. 39-De Cuyper und Urt v 26. 10.2006, C-192/05, Slg 2006, I-10451, Rdn. 31 - Tas-Hagen und Tas $\rightarrow$ Schoch JK 9/07, EGV Art. 18/1.

3 EuGH Urt v 8. 3. 2011, C-34/09, EuR 2011, 540 - Ruiz Zambrano. Weiterführend Hailbronner/Thym NJW 2011, 2008 ff.; Nettesheim JZ 2011, 1030 ff.; Graf Vitzthum EuR 2011, $550 \mathrm{ff}$.

4 EuGH Urt v 8. 3. 2011, C-34/09, EuR 2011, 540, Rdn. 39 - Ruiz Zambrano.

5 Vgl schon EuGH Urt v 20.9.2001, C-184/99, Slg 2001, I-6193, Rdn. 31 Grzelczyk $\rightarrow$ Ehlers JK 02, EGV Art. 12/1.

6 EuGH Urt v 8. 3. 2011, C-34/09, EuR 2011, 540, Rdn. 43-44 - Ruiz Zambrano.

7 EuGH Urt v 2.3.2010, C-135/08, Slg 2010, I-1449, Rdn. 42, 55 - Rottmann $\rightarrow$ Schoch JK 9/10, AEUV Art. 20/1; vgl hierzu Anmerkung von Kahl JURA 2011, $364 \mathrm{ff}$.

8 EuGH Urt v 5. 5. 2011, C-434/09, EuZW 2011, 522 - McCarthy. 
nach Unionsrecht als Unionsbürgerin bzw als Ehegatte einer Unionsbürgerin.

Der EuGH kommt zunächst - wie im Fall Ruiz Zambrano zu dem Ergebnis, dass die RL 2004/38 nicht anwendbar ist. Die doppelte Staatsangehörigkeit sei insoweit irrelevant, da sie allein nicht dazu führen könne, dass die betreffende Person automatisch so anzusehen sei, als hätte sie von ihrem Recht auf Freizügigkeit Gebrauch gemacht. In Ermangelung eines sekundärrechtlichen Aufenthaltsrechtes von Frau McCarthy verneint der EuGH konsequenterweise auch ein abgeleitetes Aufenthaltsrecht für ihren Ehemann ${ }^{9}$.

Aber auch auf der Basis von Art. 20 und 21 AEUV - also der primärrechtlichen Prüfung - besteht nach Auffassung des EuGH kein Aufenthaltsrecht. Zwar führe allein die Tatsache, dass Frau McCarthy von ihrem Recht auf Freizügigkeit keinen Gebrauch gemacht hat, nicht dazu, dass der Fall einer rein internen Situation gleichgestellt werden könne, weil im Sinne der Rechtsprechung Ruiz Zambrano jedenfalls der tatsächliche Genuss des Kernbestands der Rechte der Unionsbürgerschaft auch durch den Heimatstaat gewährt werden müsse. Jedoch werde Frau McCarthy, anders als im Fall Ruiz Zambrano, nicht verpflichtet, das Hoheitsgebiet der Union zu verlassen, da sie sich als britische Staatsangehörige weiter im Vereinigten Königreich aufhalten könne ${ }^{10}$. Dass der Ehemann der Unionsbürgerin ausgewiesen wird, scheint nach Ansicht des EuGH nicht auszureichen, um einen »Zwang « zur Ausreise wie bei den Kindern von ausreisepflichtigen Eltern anzunehmen.

Ein Abgleich der Urteile McCarthy und Ruiz Zambrano lässt also den Schluss zu, dass der Kernbereich nur in außergewöhnlichen Situationen berührt sein dürfte, insbesondere (und in der Rechtsprechung bislang ausschließlich) dann, wenn der Unionsbürger bei Verneinung eines unionsrechtlichen (derivativen) Aufenthaltsrechtes gezwungen wäre, die Union gänzlich zu verlassen. Die Berufung auf Art. 20 I AEUV kann somit nicht zu einer generellen Umgehung des grenzüberschreitenden Bezugs als Anwendungsvoraussetzung des Freizügigkeitsrechts genutzt werden, sondern greift nur in besonderen Ausnahmefällen ${ }^{11}$. Das Urteil Dereci (s. unter IV.2.) hat dieses enge Verständnis in der Folge bestätigt. Demzufolge bezieht sich das Kriterium der Verwehrung des unionsbürgerrechtlichen Kernbestandes auf Sachverhalte, »die dadurch gekennzeichnet sind, dass sich der Unionsbürger de facto gezwungen sieht, nicht nur das Gebiet des Mitgliedstaats, dem er angehört, zu verlassen, sondern das Gebiet der Union als Ganzes ${ }^{12}$.

Ein solch enges Verständnis des Ruiz Zambrano-Urteils herrscht auch an deutschen Gerichten vor. So wurde in Fällen vor dem VGH Kassel und dem VGH Mannheim keine Verletzung des Kernbestandes des Art. 20 AEUVangenommen, wenn nur der drittstaatsangehörige Vater des Kindes mit Unionsbürgerstatus ausgewiesen wurde, das Kind jedoch mit der Mutter in Deutschland bleiben konnte ${ }^{13}$. Zudem gelte auch für das Aufenthaltsrecht aus Art. 20 AEUV der Verhältnismäßigkeitsgrundsatz, sodass bei straffällig gewordenen Vätern das Aufenthaltsrecht und der Schutz des Familienlebens mit Gründen der öffentlichen Sicherheit abgewogen werden müssten ${ }^{14}$. In näherer Zukunft wird der EuGH die Frage eines Aufenthaltsrechts des Vaters im Fall Iida zu klären haben. Dieser Fall betrifft den sorgeberechtigten Vater einer minderjährigen Unionsbürgerin, die sich mit ihrer Mutter wegen deren Berufstätigkeit überwiegend in einem anderen EU-Mitgliedstaat aufhält. Der VGH Mannheim hat dem EuGH insofern die Frage vor- gelegt, ob Art. 7 und 24 GRC sowie Art. 8 EMRK eine erweiterte Auslegung der RL 2004/38/EG verlangen ${ }^{15}$. Die Entscheidung des EuGH bleibt abzuwarten.

Hinsichtlich angeheirateter drittstaatsangehöriger Ehepartner von Unionsbürgern wurde die Pflicht, trotz einer Eheschließung im EU-Ausland ein Visumverfahren durchführen zu müssen, vom BVerwG sowie den Verwaltungsgerichtshöfen Kassel und Mannheim ebenfalls nicht als Verletzung des Kernbereichs der Unionsbürgerschaft nach Art. 20 AEUV oder des Art. 21 AEUV angesehen. Jedenfalls bei Kurzaufenthalten im EU-Ausland ausschließlich zur (einfacheren) Eheschließung stelle dies keine Beschränkung des Freizügigkeitsrechts dar ${ }^{16}$. Die Unionsgrundrechte sind nach Ansicht des VGH Kassel schon nicht anwendbar, weil es sich um einen rein internen Sachverhalt handele, und überdies sei das Recht auf Familienleben durch ein Visumsverfahren nicht verletzt ${ }^{17}$.

\section{Inländerbehandlung}

Eine weitere sehr bedeutsame Entwicklung des letzten Jahrzehnts im Rahmen der Unionsbürgerschaft ist die Frage der Inländergleichbehandlung. Nach der Rechtsprechung des EuGH $^{18}$ kann sich jeder Unionsbürger, der von seinem Freizügigkeitsrecht nach Art. 21 AEUV Gebrauch gemacht hat, auf Art. 18 AEUV berufen, wonach jede Diskriminierung aus Gründen der Staatsangehörigkeit verboten ist. Daraus folgt ein Anspruch auf Inländergleichbehandlung, der in Art. 24 I der RL 2004/38 nunmehr auch ausdrücklich sekundärrechtlich verankert ist.

Im Fall Bressol ${ }^{19}$ entschied der EuGH erneut ${ }^{20}$ zu Beschränkungen des Universitätszugangs für medizinische und paramedizinische Studiengänge für ausländische Studenten. Nach der belgischen Regelung durften grundsätzlich maximal 30\% nicht ansässige Studenten zugelassen werden. Bewarben sich mehr, entschied eine Auslosung. Begründet wurde dies mit

9 Ebd, Rdn. 32-43.

10 EuGH Urt v 5. 5. 2011, C-434/09, EuZW 2011, 522, Rdn. 49-56-McCarthy. $11 \mathrm{Vgl} \mathrm{Hailbronner/Thym} \mathrm{NJW} \mathrm{2011,} 2008$ (2013).

12 EuGH Urt v 15. 11. 2011, C-256/11, NVwZ 2012, 97, Rdn. 66-Dereci u.a., mit Anmerkung von Thym NVwZ 2012, $103 \mathrm{f}$.

13 VGH Kassel Beschl v 20.10.2011, 3 A 554/11.Z, noch nicht veröffentlicht, Rdn. 16 ff., insb Rdn. 20; VGH Mannheim Urt v 7. 12. 2011, DVBl 2012, 194, Rdn. 34.

14 VGH Mannheim, Urt v 4.5.2011, NVwZ 2011, 1210, Rdn. 45-50, unter Verweis auf EuGH Urt v 2.3.2010, C-135/08, Slg 2010, I-1449, Rdn. 55 - Rottmann. Allerdings wurde vom VGH Mannheim die Verhältnismäßigkeit der Ausweisung wegen des Schutzes des Familienlebens nach Art. 8 EMRK teilweise nur aufgrund einer gleichzeitig ausgesprochenen Duldung angenommen: VGH Mannheim Urt v 7. 12. 2011, DVBl 2012, 194, Rdn. 73-78.

15 EuGH C-40/11 - Iida; vgl hierzu die Vorlage des VGH Mannheim v 20. 1. 2011, EuGRZ 2011, $96 \mathrm{ff}$.

16 BVerwG Urt v 22. 6. 2011, NVwZ 2012, 52, Rdn. 9; VGH Kassel Urt v 7. 7. 2011, 7 B 1254/11, noch nicht veröffentlicht, Rdn. 18-19; VGH Mannheim Urt v 25. 1.2010, NVwZ 2010, 529, Rdn.6. Anders wenn der Ehepartner in einem anderen Mitgliedstaat bereits über eine gültige Aufenthaltsgenehmigung nach der RL 2004/38 verfügt hat, vgl EuGH Urt v 7.7.1992, C-370/90, Slg. 1992 , I-4265, Rdn. 19-23 - Singh; Urt v 11.12.2007, C-291/05, Slg 2007, I-10719, Rdn. 35-37 - Eind

17 VGH Kassel Urt v 7.7.2011, 7 B 1254/11, noch nicht veröffentlicht, Rdn. 20-23. Vgl zum Ganzen Oberhäuser NVwZ 2012, $25 \mathrm{ff}$.

18 EuGH Urt v 20.9. 2001, C-184/99, Slg 2001, 6193, Rdn. 32-33-Grzelczyk.

19 EuGH Urt v 13.4.2010, C-73/08, Slg 2010, I-2735, insb Rdn. 66 ff. - Bressol; weiterführend insbesondere Hilpold EuZW 2010, $471 \mathrm{ff}$.

20 Ähnliche Regelungen waren bereits in einem weithin bekannten Verfahren gegen Österreich für europarechtswidrig erklärt worden, vgl EuGH Urt v 7. Juli 2005, C-147/03, Slg 2005, I-5969 - Kommission/Österreich $\rightarrow$ Ehlers JK 1/06, EGVArt. 12/3. 
dem starken Anstieg der Zahl ausländischer Studenten, insbesondere aus Frankreich, seitdem Frankreich den Zugang zu den betroffenen Studiengängen beschränkt hatte. Die französischen Studenten kehrten jedoch nach Abschluss des Studiums häufig nach Frankreich zurück, sodass in Belgien ein Mangel an (para)medizinischem Personal beklagt wurde.

Der EuGH bewertet diese Regelung als eine mittelbare Diskriminierung ${ }^{21}$, die sowohl nach Art. 24 I der RL 2004/38 als auch nach den jetzigen Art. 18 und 21 AEUV verboten sei, sofern keine Rechtfertigung greife. Allein übermäßige Belastungen zur Finanzierung des Hochschulunterrichts reichten hierfür nicht. Eine Rechtfertigung im Hinblick auf das Ziel der Sicherung der Qualität des Hochschulunterrichts und damit des Schutzes der öffentlichen Gesundheit sei indes grundsätzlich möglich. Die Prüfung des Vorliegens einer Gefahr und der Verhältnismäßigkeit überlässt der EuGH dem nationalen Gericht. Dabei sei zu berücksichtigen, dass zwischen der Ausbildung des künftigen medizinischen Personals und dem Ziel der Aufrechterhaltung einer qualitativ hochwertigen, ausgewogenen und allgemein zugänglichen medizinischen Versorgung nur ein mittelbarer Zusammenhang bestehe. Der Nachweis, dass solche Gefahren tatsächlich bestehen, müsse deshalb auf eine objektive, eingehende und auf Zahlenangaben gestützte Untersuchung gestützt sein.

Im Rahmen der Verhältnismäßigkeitsprüfung habe das nationale Gericht zum einen zu bewerten, ob eine Begrenzung der Zahl der nichtansässigen Studierenden tatsächlich geeignet ist, die Zahl der Berufsanfänger in Belgien zu erhöhen. Zum anderen sei zu prüfen, ob als milderes Mittel nicht die Möglichkeit besteht, einen Anreiz für Absolventen zu schaffen, nach Abschluss des Studiums dort zu bleiben, oder sich neu in Belgien niederzulassen. Dabei sei zu berücksichtigen, dass das Recht auf Zugang zum Hochschulunterricht zum Kernbereich des Grundsatzes der Freizügigkeit der Studierenden gehöre ${ }^{22}$.

\section{3. „Namensfälle»}

Eine wiederkehrende Fallkonstellation im Bereich der Unionsbürgerschaft sind auch die sog. Namensfälle. In den Rechtssachen Sayn-Wittgenstein ${ }^{23}$ und Runevic-Vardyn ${ }^{24}$ schließt der EuGH an die Fälle Garcia Avello ${ }^{25}$ und Grunkin und $P_{a u l}{ }^{26}$ an, welche die Nutzung des Doppelnamens für Kinder mit doppelter Staatsangehörigkeit bzw Wohnsitz in einem anderen Mitgliedstaat betrafen. Diesmal entscheidet er jedoch gegen die betroffenen Unionsbürger und gewährt den Mitgliedstaaten gewisse Spielräume.

\section{a) Urteil Sayn-Wittgenstein}

Im Fall Sayn-Wittgenstein hatte sich eine in Deutschland sesshafte österreichische Staatsangehörige im Erwachsenenalter von einem deutschen Adeligen adoptieren lassen, um den Adelstitel »Fürstin von Sayn-Wittgenstein « zu erlangen. Dieser wurde 1992 in das österreichische Geburtenbuch eingetragen. Die zuständige österreichische Behörde wollte den Adelszusatz (»Fürstin von«) jedoch im Jahre 2007 löschen, weil der österreichische Verfassungsgerichtshof in einer Entscheidung im Jahre 2003 festgestellt hatte, dass es gegen das - im Verfassungsrang stehende und den republikanischen Gleichheitsgrundsatz durchsetzende - Adelsaufhebungsgesetz verstoße, wenn ein österreichischer Staatsbürger im Wege der Adoption einen Familiennamen erwirbt, der einen früheren Adelstitel enthält. Da Frau Sayn-Wittgenstein sich bereits seit geraumer Zeit unter diesem Adelsnamen im europäischen
Ausland aufhielt, stellte sich die Frage, ob der Löschung des Namens Art. 21 AEUV entgegensteht.

Der EuGH nimmt eine Beschränkung des Art.21 AEUV durch die (geplante) Löschung des Adelstitels aus dem österreichischen Geburtenbuch an. Er rekapituliert zunächst die eingangs genannten Vorgängerentscheidungen zur DoppelnamenProblematik, wonach eine Beschränkung von Art. 21 AEUV u. a. daraus folgen könne, dass der Heimatstaat bestimmte Nachteile an die Ausübung der Freizügigkeit in einem anderen EU-Staat knüpft. Wann ein solcher »Nachteil« vorliegt, beurteilt der EuGH im Lichte der Art. 7 GRC und 8 EMRK, die den Namen als Teil der individuellen Selbstbestimmung schützen. Der vorliegende Fall unterscheidet sich von den bisherigen Namensfällen vor allem dadurch, dass Frau Sayn-Wittgenstein zum einen keine doppelte Staatsangehörigkeit besaß und ihr Name zum anderen allein im österreichischen Geburtenbuch eingetragen war. Nach Auffassung des Gerichtshofs liegt ein »schwerwiegender Nachteil « der (geplanten) Namenslöschung allerdings darin, dass Frau Sayn-Wittgenstein 15 Jahre lang vor allem in einem anderen Mitgliedstaat unter dem Adelstitel aufgetreten war und die förmlichen Spuren dieses Namens (Führerschein, Konto, Krankenkasse etc.) umfänglich zu tilgen habe, um den Verdacht von Falschangaben zu verhindern ${ }^{27}$.

Allerdings sieht der EuGH die Beschränkung als gerechtfertigt an. Hier entfaltet das Urteil erst seine eigentliche Tragweite. Denn es handelt sich um das erste Urteil überhaupt, in dem der Gerichtshof die durch den Vertrag von Lissabon reformulierte und nunmehr justiziable Klausel zum Schutz der nationalen Identität in Art. 4 II EUV heranzieht. Der EuGH folgt der Argumentation Österreichs, wonach das der Durchsetzung des republikanischen Gleichheitssatzes dienende Adelsaufhebungsgesetz Bestandteil der »nationalen Identität « Österreichs sei. Die Berufung auf diese verfassungsrechtliche Sondersituation ordnet der Gerichtshof sodann dogmatisch dem Rechtfertigungsgrund der öffentlichen Ordnung $\mathrm{zu}^{28}$. Auch wenn dieser letzterer eng auszulegen sei und tatbestandlich eine tatsächliche und hinreichend schwere Gefährdung voraussetze, billigt der EuGH den Mitgliedstaaten gleichwohl insoweit einen Beurteilungsspielraum zu, als die konkreten Umstände, die möglicherweise die Berufung auf den Begriff der öffentlichen Ordnung rechtfertigen, von einem Mitgliedstaat zum anderen und im zeitlichen Wechsel verschieden sein können ${ }^{29}$. Nach der knappen Feststellung, dass der Schutz des Gleichheitssatzes schon mit Blick auf Art. 20 GRC aus unionsrechtlicher Sicht als legitimer Zweck einzustufen sei, unterstreicht der EuGH im Rahmen der Prüfung der Erforderlichkeit sodann, dass die Verhältnismäßigkeit nicht bereits daran scheitere, dass ein Mit-

21 Mittelbar deshalb, weil sie nicht unmittelbar an die Staatsangehörigkeit, sondern an das Ansässigkeitserfordernis anknüpft.

22 EuGH Urt v 13. 4. 2010, C-73/08, Slg 2010, I-2735, insb Rdn. 66 ff. - Bressol.

23 EuGH Urt v 22. 12. 2010, C-208/09, EuGRZ 2011, 25 - Sayn-Wittgenstein.

24 EuGH Urt v 12.5.2011, C-391/09, noch nicht veröffentlicht - Runevic-Vardyn.

25 EuGH Urt v 2. 10. 2003, C-148/02, Slg 2003, I-11613 - Garcia Avello.

26 EuGH Urt v 14.10.2008, C-353/06, Slg 2008, I-7639 - Grunkin und Paul $\rightarrow$ Ehlers JK 5/09, EGV Art. 18/3.

27 EuGH Urt v 22. 12. 2010, C-208/09, EuGRZ 2011, 25, Rdn. 66-71 - Sayn-Wittgenstein.

28 Ebd, Rdn. 83-84.

$29 \mathrm{Vgl}$ in diesem Sinne bereits hinsichtlich der Grundfreiheiten EuGH Urt v 4. 12.1974, Rs 41/74, Slg 1974, 1337, Rdn. 18/19 - van Duyn, sowie Urt v 27. 10.1977, Rs 30/77, Slg 1977, 1999, Rdn.33/35 - Bouchereau, und Urt v 14. 10. 2004, Rs C-36/02, Slg 2004, I-9609, Rdn. 31 - Omega $\rightarrow$ Ehlers JK 6/05, EGV Art. 49/13. 
gliedstaat andere Schutzregelungen erlassen hat als ein anderer. An dieser Stelle rekurriert der Gerichtshof auf Art. 4 II EUV und lässt - freilich innerhalb des durch das Unionsrecht gezogenen Rahmens - die spezifische verfassungsrechtliche Wertung Österreichs durchschlagen ${ }^{30}$. Der EuGH führt damit einen Ansatz identitätsschonender Rechtsprechung fort, den er bereits zuvor im Urteil Omega gewählt, damals aber nicht ausdrücklich auf die (zu diesem Zeitpunkt noch nicht justiziable Vorläufernorm der) Identitätsklausel bezogen hatte.

\section{b) Urteil Runevič-Vardyn}

Ebenfalls auf die nationale Identität Bezug nahm der EuGH im Fall Runevič-Vardyn zur litauischen Schreibweise polnischer Namen. Die litauische Staatsangehörige Frau Malgožata Runevič-Vardyn gehört einer polnischen Minderheit an. Ihr polnischer Name »Malgorzata Runiewičz« wurde von den litauischen Behörden in litauischer Schreibform eingetragen, nämlich als »Malgožata Runevič«. Nachdem sie eine gewisse Zeit in Polen gewohnt und gearbeitet hatte, heiratete sie den polnischen Staatsangehörigen Łukasz Paweł Wardyn. Dessen Name wurde in der litauischen Heiratsurkunde in »Lukasz Pawel Wardyn « ohne das diakritische $€$ geschrieben. Der Name der Ehefrau wurde dagegen in der litauischen Schreibweise als »Malgožata Runevič-Vardyn « angegeben. Wohnsitz der Ehegatten zum Entscheidungszeitpunkt war Belgien. Frau Runevič-Vardyn beantragte beim litauischen Standesamt, den in ihrer Geburtsurkunde eingetragenen Namen in »Malgorzata Runiewicz « und den in ihrer Heiratsurkunde eingetragenen Namens in »Malgorzata Runiewicz-Wardyn « zu ändern. Herr Wardyn wollte seine Vornamen in der Heiratsurkunde mit dem diakritischen » $€$ « geschrieben haben. Dies wurde von den litauischen Behörden abgelehnt.

Der Gerichtshof hält einen Verstoß gegen Art. 18 und 21 AEUV nur hinsichtlich der Schreibweise des Namens »Vardyn « im Namen von Frau Runevič-Vardyn für möglich, lehnt im Übrigen aber einen Verstoß gegen Unionsrecht ab. Zwar sei es mit dem Recht auf Freizügigkeit unvereinbar, wenn der Heimatstaat einen Unionsbürger deshalb weniger günstig behandele, weil er von seinem Freizügigkeitrecht Gebrauch gemacht habe $^{31}$. Auch seien der Vor- und der Nachname einer Person Teil ihrer Identität und ihres Privatlebens, deren Schutz, wie gesehen auch durch die GRC und die EMRK gewährleistet werde $^{32}$. Hinsichtlich der Schreibung des Vor- und Mädchennamens in der litauischen Geburtsurkunde und in der litauischen Heiratsurkunde von Frau Runevič-Vardyn liege jedoch kein Verstoß gegen Unionsrecht, weil sie insofern von ihrem Heimatstaat Litauen nicht schlechter behandelt werde als vor dem Gebrauch der Freizügigkeit ${ }^{33}$.

In Bezug auf den Antrag, in der litauischen Heiratsurkunde den dem Mädchennamen der Ehefrau hinzugefügten zweiten Nachnamen in Wardyn zu ändern, schließt der Gerichtshof dagegen nicht aus, dass deren Verweigerung »schwerwiegende Nachteile « administrativer, beruflicher und privater Art bewirken könne ${ }^{34}$. Solche Nachteile könnten sich aus der abweichenden Umschrift ein- und desselben Nachnamens von Eheleuten ergeben. Zwar könne diese Beeinträchtigung durch das Ziel, die offizielle Landessprache zu schützen, gerechtfertigt werden. Nach Art. 3 III UA 4 EUV und Art. 22 der GRC wahrt die Union den Reichtum ihrer kulturellen und sprachlichen Vielfalt und gemäß Art. 4 II EUV achtet die Union die nationale Identität ihrer Mitgliedstaaten, zu der auch der Schutz der offiziellen Landessprache gehöre. Dabei müsse jedoch ein angemessenes Gleichgewicht zwischen dem Recht der Ehegatten auf Achtung ihres Privat- und Familienlebens und dem legitimen Schutz der offiziellen Landessprache und der Traditionen des Mitgliedstaats gewahrt werden. Es könne deshalb unverhältnismäßig sein, im vorliegenden Fall keine Ausnahme zuzulassen, insbesondere wenn in anderen Fällen wie z. B. bei Ausländern ebenfalls Ausnahmen gemacht werden ${ }^{35}$.

Zum Antrag des Ehemanns, Herrn Wardyn, seine Vornamen mit dem diakritischen » $€$ « umzuschreiben, verneint der Gerichtshof einen Verstoß gegen Unionsrecht, da diese Zeichen bei vielen Handlungen des täglichen Lebens aus technischen Gründen (wie z. B. objektiven Zwängen bestimmter Informatiksysteme) weggelassen würden und außerdem eine Person, die keine Fremdsprache beherrsche, die Bedeutung dieser Zeichen oftmals nicht kenne. Es sei daher wenig wahrscheinlich, dass dem Betroffenen allein durch das Weglassen dieser Zeichen tatsächliche und schwerwiegende Nachteile erwachsen könnten, die geeignet wären, Zweifel an seiner Identität und an der Echtheit der von ihm vorgelegten Dokumente zu wecken ${ }^{36}$.

Als Ergebnis dieser Rechtsprechung lässt sich feststellen, dass der EuGH nationale Besonderheiten, sei es bei der Behandlung von Adelstiteln, sei es bei der Schreibweise der Landessprache, nunmehr deutlicher als bisher Ernst nimmt und sie als Teil der nationalen Identität im Sinne von Art. 4 II EUV anerkennt. Gleichzeitig räumt er den Mitgliedstaaten insofern kein umfassendes Ermessen ein, sondern überprüft auch im Fall solcher nationaler Gestaltungsspielräume, ob den Unionsbürgern durch die nationalen Regelungen schwerwiegende Nachteile entstehen und ob diese nicht durch Ausnahmeregelungen verhindert werden können.

\section{Ausweisung aus Gründen der öffentlichen Sicherheit}

Im Rahmen des allgemeinen Freizügigkeitsrechts für die Ausbildung ebenfalls relevant sind die bereits erwähnte Freizügigkeits- und Aufenthaltsrichtlinie 2004/38 und die hierzu ergangene Rechtsprechung. Im Fall Tsakouridis ${ }^{37}$ konkretisierte der EuGH auf der Basis der ständigen Rechtsprechung die Ausweisungsgründe der öffentlichen Ordnung und Sicherheit, die sich ursprünglich für Arbeitnehmer aus dem heutigen Art. 45 III AEUV sowie den speziellen Freizügigkeitsrichtlinien ergaben und die nunmehr in Art. 27 und 28 RL 2004/38 kodifiziert sind. Das Urteil eignet sich daher sehr gut für die Wiederholung dieser Materie.

Herr Tsakouridis, ein griechischer Staatsangehöriger, der in Deutschland aufgewachsen war und lebte, wurde mehrfach wegen Körperverletzung und anderer Delikte verurteilt. Von März 2004 bis Mitte Oktober 2004 betrieb er einen Crêpes-Stand in Griechenland. Mitte Oktober 2005 ging Herr Tsakouridis wieder nach Rhodos. Kurz darauferließ das Amtsgericht Stuttgart einen internationalen Haftbefehl gegen ihn wegen Drogendelikten. Er wurde auf Rhodos festgenommen, nach Deutschland überführt

30 EuGH Urt v 22. 12. 2010, C-208/09, EuGRZ 2011, 25, Rdn. 90-95 - Sayn-Wittgenstein.

31 Vgl schon EuGH Urt v 11.7.2002, C-224/98, Slg 2002, I-6191, Rdn. 30 $D<$ Hoop.

32 EuGH Urt v 12. 5. 2011, C-391/09, noch nicht veröffentlicht, Rdn. 66 - Runevic-Vardyn.

33 Ebd, Rdn. 69-71.

34 Vgl schon EuGH Urt v 2.10.2003, C-148/02, Slg 2003, I-11613, Rdn. 36 Garcia Avello; Urt v 22. 12. 2010, C-208/09, EuGRZ 2011, 25, Rdn. 55, 66-70 - Sayn-Wittgenstein.

35 EuGH Urt v 12.5. 2011, C-391/09, Rdn. 75-92 - Runevic-Vardyn.

36 Ebd, Rdn. 81-82

37 EuGH Urt v 23. 11. 2010, C-145/09, NVwZ 2011, 221 - Tsakouridis. 
und zu einer Strafe von sechs Jahren verurteilt. 2008 stellte die zuständige Behörde den Verlust seines Aufenthaltsrechts fest und drohte die Abschiebung nach Griechenland an. Sie begründete dies mit der Gefährdung der öffentlichen Ordnung. Die Integration in Griechenland sei nicht gefährdet.

Die Ausweisung von Unionsbürgern ist in Art. 27 und $28 \mathrm{RL}$ 2004/38 geregelt. Besonderer Ausweisungsschutz nach der Richtlinie wird bei einem Aufenthalt von mehr als fünf Jahren gewährt. Nach zehn Jahren darf eine Ausweisung nur noch aus zwingenden Gründen der öffentlichen Sicherheit erfolgen. Hinsichtlich der Frage, ob Herr Tsakouridis unter diesen besonderen Ausweisungsschutz fallen sollte, stellte sich zunächst die Frage, wie seine Abwesenheit in Deutschland zu beurteilen ist. Der EuGH verlangt insofern, alle im Einzelfall relevanten Umstände zu berücksichtigen, insbesondere die Dauer jeder einzelnen Abwesenheit des Betroffenen, die Gesamtdauer und die Häufigkeit der Abwesenheiten sowie die Gründe dafür, anhand derer festzustellen ist, ob der Betroffene den Mittelpunkt seiner persönlichen, familiären oder beruflichen Interessen bereits in einen anderen Mitgliedstaat verlagert hat. Die Entscheidung im Einzelfall überlässt der EuGH dem nationalen Gericht, jedoch müsse auch die zwangsweise Rückführung und die Zeit im Gefängnis Berücksichtigung finden ${ }^{38}$.

Hinsichtlich der Frage, ob ein Ausweisungsgrund aufgrund schwerwiegender bzw zwingender Gründe der öffentlichen Sicherheit und Ordnung gegeben sei, bezog sich der EuGH bei der Auslegung der Richtlinienbestimmungen auf die ständige Rechtsprechung seit dem Urteil Bouchereau ${ }^{39}$, die in Art. 27 und 28 nunmehr konkretisiert ist. Zudem ist nach Ansicht des EuGH das Ziel der Richtlinie zu berücksichtigen, den Schutz gut integrierter Unionsbürger vor Ausweisung zu stär$\mathrm{ken}^{40}$. Deshalb seien nur Beeinträchtigungen eines Grundinteresses der Gesellschaft von einem besonders hohen Schweregrad und somit außergewöhnliche Umstände umfasst. Der illegale Drogenhandel wird vom EuGH als eine Bedrohung der Gesundheit, Sicherheit und Lebensqualität der Unionsbürger sowie der legalen Wirtschaftstätigkeit, der Stabilität und der Sicherheit der Mitgliedstaaten angesehen, weshalb die Bekämpfung der mit dem bandenmäßigen Handel mit Betäubungsmitteln verbundenen Kriminalität einen »schwerwiegenden« und sogar einen »zwingenden Grund der öffentlichen Sicherheit « darstelle ${ }^{41}$.

Allerdings sei auch bei Vorliegen schwerwiegender oder zwingender Gründe eine individuelle Prüfung des Einzelfalls erforderlich. Das persönliche Verhalten der betreffenden Person müsse eine tatsächliche und gegenwärtige Gefahr darstellen. Vorangegangene strafrechtliche Verurteilungen allein reichten nicht aus und vom Einzelfall losgelöste oder auf Generalprävention verweisende Begründungen seien nicht zulässig. Das nationale Gericht müsse die verhängten Strafen, den Grad der Beteiligung an der kriminellen Aktivität, den Umfang des Schadens und die Rückfallneigung berücksichtigen und gegen die Gefahr für die Resozialisierung des Unionsbürgers in dem Staat, in den er vollständig integriert ist, abwägen. Bei dieser Abwägung seien gerade auch die Grundrechte zu beachten, insbesondere das Recht auf Achtung des Privat- und Familienlebens gemäß Art. 7 GRC und Art. 8 der EMRK. Im Fall eines Unionsbürgers, der die meiste oder die gesamte Zeit seiner Kindheit und Jugend rechtmäßig im Aufnahmemitgliedstaat verbracht habe, müssten sehr stichhaltige Gründe vorgebracht werden, um die Ausweisungsmaßnahme zu rechtfertigen ${ }^{42}$.

Dieses Urteil ist damit ein sehr anschauliches Beispiel für die strikte Verhältnismäßigkeitsprüfung, die der EuGH bei der
Ausweisung von Unionsbürgern vornimmt. Der Fall kann sowohl in Bezug auf das Primär- wie auch das Sekundärrecht konstruiert werden und ist daher sehr prüfungsrelevant.

Interessant ist auch die Übertragbarkeit dieser Grundsätze auf türkische Staatsangehörige. Da diese keine Unionsbürger sind und das Assoziierungsabkommens EWG-Türkei lediglich auf wirtschaftliche Integration gerichtet ist, kann insofern nach dem Urteil Ziebell der besondere Ausweisungsschutz der RL 2004/38 nicht entsprechend angewendet werden ${ }^{43}$. Jedoch gelten bei der Auslegung der Gründe der öffentlichen Ordnung und Sicherheit iSv Art. 14 I des Assoziationsratsbeschlusses Nr.1/80 die in der Rechtsprechung zu Arbeitnehmern seit Bouchereau entwickelten Grundsätze, insb das Erfordernis einer tatsächlichen und gegenwärtigen Gefahr. Außerdem sind analog zu Art. 12 III der RL 2003/109/EG (betreffend die Rechtsstellung der langfristig aufenthaltsberechtigten Drittstaatsangehörigen) die Aufenthaltsdauer, das Alter, die Folgen einer Ausweisung für die betreffende Person und ihre Familienangehörigen sowie ihre Bindungen zum Aufenthaltsstaat oder fehlende Bindungen zum Herkunftsstaat zu berücksichtigen ${ }^{44}$.

Schließlich ist noch anzumerken, dass diese Grundsätze, wie im Fall Gaydarov bestätigt, auch gegenüber dem Heimatstaat gelten, wenn dieser die Bewegungsfreiheit eigener Staatsangehöriger wegen deren Straftaten in anderen Staaten einschränken möchte ${ }^{45}$. Zudem hat der Unionsbürger Anspruch auf einen wirksamen gerichtlichen Rechtsbehelf sowie $\mathrm{zu}$ diesem Zweck auf eine Begründung der staatlichen Maßnahme ${ }^{46}$.

\section{Grundfreiheiten}

Nach wie vor stammen die mit Abstand meisten europarechtlichen Prüfungsaufgaben aus dem Bereich der Grundfreiheiten. Aktuelle Rechtsprechungsentwicklungen sollten in diesem Bereich besonders genau verfolgt und nachbereitet werden.

\section{Allgemeine Entwicklungen}

In der Rechtsprechung der vergangenen zweieinhalb Jahre zeichnen sich einige allgemeine Trends ab, die vorangestellt werden, bevor auf die einzelnen Grundfreiheiten eingegangen wird.

\section{a) Das Kriterium des Marktzugangs im Rahmen der Beschränkung}

Ein Prüfungsklassiker, der nicht nur für den Bereich der Warenverkehrsfreiheit relevant ist, ist seit jeher die Frage, welche Kriterien der Annahme einer Beschränkung und damit der Eröffnung des Anwendungsbereiches der betreffenden Grundfreiheit

38 Ebd, Rdn. 32-35.

39 EuGH Urt v 27. 10. 1977, 30/77, Slg 1977, 1999, Rdn. 29 - Bouchereau.

40 23. und 24. Erwägungsgrund der RL 2004/38.

41 EuGH Urt v 23. 11. 2010, C-145/09, NVwZ 2011, 221, Rdn. 43-48 - Tsakouridis.

42 Ebd, Rdn. 49-54

43 EuGH Urt v 8. 12. 2011, C-371/08, noch nicht veröffentlicht, Rdn. 57 ff., insb 68-73- Ziebell.

44 Ebd, Rdn. 79-83, vgl insofern auch die hierzu ergangene Rechtsprechung des VGH Mannheim, etwa kürzlich: Urt v 7.3.2012, 11 S 3269/11, noch nicht veröffentlicht, Rdn. $39 \mathrm{ff}$.

45 EuGH Urt v 17. 11. 2011, C-430/10, EuZW 2012, 72, Rdn. 24-26 - Gaydarov; vgl auch EuGH Urt v 10. 7. 2008, C-33/07, Slg 2008, I-5173, Rdn. 17-20 - Jipa.

46 EuGH Urt v 17. 11.2011, C-430/10, EuZW 2012, 72, Rdn. 41 - Gaydarov, vgl hierzu schon EuGH Urt v 15. 10. 1987, Rs 222/86, Slg 1987, 4097, Rdn. 14-15Heylens u.a. 
zugrundezulegen sind. Auch wenn die Übertragbarkeit der Keck-Rechtsprechung ${ }^{47}$ auf andere Grundfreiheiten weiterhin stark umstritten bleibt ${ }^{48}$, hat das im Zuge dieser Rechtsprechung herausgestellte Kriterium der Beeinträchtigung des Marktzugangs verstärkten Eingang in die Rechtsprechung des Gerichtshofs auch $\mathrm{zu}$ anderen Grundfreiheiten gefunden ${ }^{49}$. Wie wenig konkretisierend (und limitierend) dieses Kriterium allerdings ist, illustriert das Urteil Kfz-Haftpflichtversicherung ${ }^{50}$. Darin ging es um einen unterschiedslos anwendbaren Kontrahierungszwang und eine Angemessenheitskontrolle für die Prämienhöhe.

Unter Verweis auf seine Rechtsprechung in der Rechtssache CaixaBank France ${ }^{51}$, in der es um das Verbot verzinster Girokonten ging, wendet der EuGH als Obersatz zunächst die Gebhard-Formel an, wonach unter den Begriff der Beschränkung alle Maßnahmen fallen, welche die Ausübung der Niederlassungsfreiheit oder des freien Dienstleistungsverkehrs verbieten, behindern oder weniger attraktiv machen. Daran anknüpfend erinnert der EuGH allerdings daran, dass unterschiedslos anwendbare Maßnahmen nichtbereits deshalbeine Beschränkung darstellten, weil andere Mitgliedstaaten die in ihrem Gebiet ansässigen Erbringer gleichartiger Dienstleistungen weniger strengen oder wirtschaftlich interessanteren Vorschriften unterwerfen. Eine Beschränkung umfasse aber jedenfalls »die von einem Mitgliedstaat getroffenen Maßnahmen, die, obwohl sie unterschiedslos anwendbar sind, den Marktzugang von Unternehmen aus anderen Mitgliedstaaten betreffen und somit den innergemeinschaftlichen Handel behindern ${ }{ }^{52}$. In der Subsumtion stellt der EuGH fest, dass zwar die Zulassung der Versicherer unberührt bleibe, dass der Kontrahierungszwang jedoch eine erhebliche Einmischung in die den Wirtschaftsteilnehmern grundsätzlich zustehende Vertragsfreiheit darstelle. Verbunden mit der Pflicht zur maßvollen Tarifgestaltung stelle dies eine erhebliche Belastung dar, die die Geschäftsstrategie der Versichererbeeinflusse, den Marktzugang beeinträchtige und letztlich die Ausübung der Grundfreiheiten weniger attraktiv mache ${ }^{53}$.

Auch im Urteil Kommission/Spanien ${ }^{54}$ zur städtebaulichen Begrenzung und zur Genehmigungspflicht von neuen großen Einzelhandelsgeschäften zeigt sich die Weite des Kriteriums der Behinderung des Marktzugangs. Unabhängig davon, dass die Genehmigungspflicht ebenso spanische Unternehmen betrifft, macht sie nach Ansicht des EuGH die Ausübung der Niederlassungsfreiheit auf dem spanischen Markt weniger attraktiv: Die Verkaufsflächen insgesamt werden in weniger besiedelten Gebieten begrenzt, die Regelungen für bestehende Einrichtungen gelten nicht und die Verfahrensregelungen sowie die anzuwendenden Kriterien sind so strikt, dass voraussichtlich Anträge abgelehnt werden ${ }^{55}$.

Damit ist festzustellen, dass derEuGHimmerhäufigerauch im Bereich der Dienstleistungs- und Niederlassungsfreiheit sowie der Arbeitnehmerfreizügigkeit auf die marktzugangsbeschränkende Wirkung Bezug nimmt, um eine Beschränkung iSd Gebhard-Formel zu bejahen. Dabei geht es bemerkenswerterweise nicht um eine »Rückausnahme« wie mit der Keck-Formel sondern um die positive Feststellung der Beschränkung selbst. Eine entsprechende Entwicklung könnte sich seit der Entscheidung Kommission/Italien (Krad-Anhänger) auch für die Warenverkehrsfreiheit abzeichnen (dazu sogleich unter II.2.b.).

\section{b) Das Kriterium »zu ungewiss und indirekt"}

Zur Einschränkung des weiten Beschränkungsbegriffs hat der EuGH neben der Keck-Formel und anderen Strategien auch eine Formel entwickelt, nach der die Beeinträchtigung des Han- dels nicht zu ungewiss und indirekt sein darf. Dies geschah zunächst für die Warenverkehrsfreiheit ${ }^{56}$, in weiteren Fällen aber auch für die Niederlassungsfreiheit ${ }^{57}$, die Arbeitnehmerfreizügigkeit ${ }^{58}$ und das allgemeine Freizügigkeitsrecht ${ }^{59}$ sowie nunmehr auch für die Dienstleistungsfreiheit ${ }^{60}$. Die betreffenden Regelungen unterschieden nicht nach dem Ursprung der beförderten Waren und sollten nicht den Handel mit anderen Mitgliedstaaten regeln. Der EuGH sah die Behinderung des Handels entweder als unwahrscheinlich oder als eigentlich durch andere Faktoren verursacht an. Ihre Wirkungen befand der EuGH deshalb als » zu ungewiss und zu indirekt «, um den Handel zwischen den Mitgliedstaaten zu behindern. Diese Ausnahme wird in der Literatur teilweise gleichwohl darauf reduziert, sie solle rein hypothetische Kausalverläufe, bei denen die handelsbeschränkende Wirkung nicht ersichtlich oder jedenfalls vom Mitgliedstaat nicht hinreichend dargetan sei, vom Tatbestand ausnehmen ${ }^{61}$. Die Rechtsprechung knüpft jedoch nicht

47 EuGH Urt v 24. 11. 1993, verb Rs C-267/91 und C-268/91, Slg 1993, I-6097, insb Rdn. 14-17 - Keck und Mithouard.

$48 \mathrm{Vgl} \mathrm{dazu}$ (bejahend) Ehlers in: ders (Hrsg), Europäische Grundrechte und Grundfreiheiten, 3. Aufl 2009, §7 Rdn. 87 m. w. N.

49 Vgl nur für die Arbeitnehmerfreizügigkeit das Urt v 15. 12. 1995, C-415/93, Slg 1995, I-4921, Rdn. 103 - Bosman; für die Dienstleistungsfreiheit das Urt v 10.5.1995, C-384/93, Slg 1995, I-1141, Rdn.36-38 - Alpine Investments; für die Niederlassungsfreiheit das Urt v 5. 10. 2004, C-442/02, Slg 2004, I-8961, Rdn. 12-16 - CaixaBank France.

50 EuGH Urt v 28.4.2009, C-518/06, Slg 2009, I-3491, Rdn.62-70 - Kommission/Italien (Kfz-Haftpflichtversicherung).

51 EuGH Urt v 5. 10. 2004, C-442/02, Slg 2004, I-8961, Rdn. 12-16-CaixaBank France.

52 EuGH Urt v 28. 4. 2009, C-518/06, Slg 2009, I-3491, Rdn. 64-Kfz-Haftpflichtversicherung.

53 Anders als in CaixaBank France sieht er die Beschränkung jedoch als gerechtfertigt an, nämlich erforderlich um die Einhaltung der Versicherungspflicht und damit das Ziel, die Entschädigung von Verkehrsunfallopfern zu garantieren, zu erreichen; ebd, Rdn. 72-93.

54 EuGH Urt v 24.3. 2011, C-400/08, EuZW 2011, 557 - Kommission/Spanien (Einzelhandelsgeschäfte); mit Anmerkung von Wiggers NJW-Spezial 2011, $556 \mathrm{f}$.

55 EuGH Urt v 24.3.2011, C-400/08, EuZW 2011, 557, Rdn. 64-72 - Kommission/Spanien (Einzelhandelsgeschäfte). Zwar wird die Genehmigungspflicht grundsätzlich als gerechtfertigt angesehen. Eine Rechtfertigung der Begrenzung von Verkaufsflächen zur Vermeidung eines Überangebots aus Gründen der Raumplanung, des Umweltschutzes wegen der Vermeidung von unnötigem Verkehr zu den Einkaufszentren und des Verbraucherschutzes wird jedoch abgelehnt, weil insgesamt die Möglichkeiten zur Errichtung der Einkaufseinrichtungen eingeschränkt würden und es sich um rein wirtschaftliche Erwägungen handele, ebd, Rdn. 82-84, 89.

56 EuGH Urt v 7.3.1990, C-69/88, Slg I-583, Rdn. 11 - Krantz (Befugnis des Fiskus zur Pfändung von unter Eigentumsvorbehalt verkauften Sachen); Urt v 13.10.1993, C-93/92, Slg 1993, I-5009, Rdn. 12 - CMC Motorradcenter (Aufklärungspflichten); Urt v 14. 7. 1994, C-379/92, Slg 1994, I-3453, Rdn. 24 - Peralta (Verbot der Einleitung schädlicher Chemikalien); Urt v 18. 6. 1998, C-266/96, Slg 1998, I-3949, Rdn. 31 - Corsica Ferries France (Pflicht zur Inanspruchnahme von örtlichen Festmachgruppen im Schiffsverkehr). Urt v 22.6.1999, C-412/97, Slg 1999, I-3845, Rdn. 11 - ED (Nichtdurchführung eines Mahnverfahrens).

57 EuGH Urt v 20.6. 1996, verb Rs C-418/93 u. a., Slg 1996, I-2975, Rdn. 32 Semeraro Casa Uno Srl u. a. (Ladenschlussregelungen). Abgelehnt dagegen in EuGH Urt v 1.4.2008, C-212/06, Slg 2008, I-1683, Rdn. 51-52 - Flämische Pflegeversicherung.

58 EuGH Urt v 27.1.2000, C-190/98, Slg 2000, I-493, Rdn. 25 - Graf (kein Abfindungsanspruch bei Kündigung seitens des Arbeitnehmers).

59 EuGH Urt v 23. 10. 2010, verb Rs C-11/06 u. a., Slg 2007, I-9161, Rdn. 32 Morgan und Bucher $\rightarrow$ Schoch JK 5/08, EGV Art. 18/2.

60 EuGH Urt v 15.6.2010, C-211/08, Slg 2010, I-5267, Rdn. 68-73 - Kommission/Spanien (Krankenhausbehandlung); vgl aber auch schon Urt v 17. 2. 2005, C-134/03, Slg 2005, I-1167, Rdn. 38 - Viacom Outdoor.

61 Epiney in: Ehlers (Hrsg), Europäische Grundrechte und Grundfreiheiten, 3. Aufl 2009, § 8 Rdn.50; Kingreen in: Calliess/Ruffert (Hrsg), EUV/AEUV, 
allein an fehlende Darlegungen der Mitgliedstaaten oder Ungewissheit an, sondern an die konkreten Auswirkungen der Maßnahme selbst. Die Wirkung und Wirkungsintensität und damit die Spürbarkeit der Maßnahme im grenzüberschreitenden Verkehr scheint daher eine Rolle zu spielen. Faktisch könnte dies auf eine Art de minimis-Ausnahme hinauslaufen, als welche diese Rechtsprechung teilweise auch angesehen wird ${ }^{62}$. Andererseits wiederholt der EuGH in anderen Entscheidungen, dass ein Nachweis, dass sich die Maßnahme spürbar auf diesen Handel ausgewirkt haben müsse, nicht erforderlich sei, und lässt daher auch unbedeutende Auswirkungen genügen ${ }^{63}$. Eine genaue Einordnung dieses Kriteriums und eine Konkretisierung seines Inhalts stehen damit noch aus.

Dennoch wendet der EuGH die Formel der »zu ungewissen und indirekten « Beeinträchtigung in seiner jüngsten Rechtsprechung häufiger an, etwa in der Rechtssache Guarnieri ${ }^{64}$, die eine Regelung betraf, welche die Leistung einer Prozesskostensicherheit vor belgischen Zivilgerichten nur für Ausländer vorsah. Im Rahmen der Prüfung von Art. 34 AEUV und der Dassonville-Formel stellt der EuGH fest, dass die Regelung rein verfahrensrechtlicher Art und nicht darauf gerichtet sei, den Warenverkehr zu regulieren. Ihre Anwendung hänge nicht von der Herkunft des in Rede stehenden Erzeugnisses, sondern von zwei kumulativen Voraussetzungen ab, die erst nach dem Zugang des Produkts zum belgischen Markt hinzutreten: Zum einen müsse ein Rechtsstreit nach Abschluss eines Vertrags entstehen und $\mathrm{zu}$ einer Klage vor den belgischen Gerichten führen; zum anderen müsse der Beklagte in einem solchen Verfahren ein belgischer Staatsangehöriger sein, der sich dafür entscheide, sich auf die betreffende Bestimmung $\mathrm{zu}$ berufen. Der Umstand, dass Bürger eines anderen Mitgliedstaats aus diesem Grund zögern würden, Waren an Kunden in diesem Mitgliedstaat zu verkaufen, die dessen Staatsangehörigkeit besitzen, sei zu ungewiss und zu mittelbar, als dass eine solche nationale Maßnahme als geeignet angesehen werden könne, den innergemeinschaftlichen Handel zu behindern.

Auch in dem Urteil Kommission/Spanien ${ }^{65}$ zur Kostentragung nach einer ungeplanten Krankenhausbehandlung im Ausland lehnte der EuGH eine Beeinträchtigung der Dienstleistungsfreiheit ab, weil die Auswirkungen der Regelung auf die Wahl eines Krankenhauses im Ausland oder im Inland zu ungewiss und mittelbar und daher nicht geeignet seien, die Dienstleistungsfreiheit zu behindern (siehe unten unter II.5.b.).

In anderen Fällen bejaht der EuGH den Tatbestand der betreffenden Grundfreiheit aber auch, obwohl fraglich erscheint, dass die Regelung im konkreten Fall eine Person von der Ausübung ihrer Freizügigkeit abhalten könnte. So entschied der EuGH im Fall der Flämischen Pflegeversicherung ${ }^{66}$, dass das Erfordernis eines Wohnsitzes in der betreffenden Region in Belgien für die Zugehörigkeit zur staatlichen Pflegeversicherung geeignet sei, die Ausübung der Niederlassungsfreiheit und der Arbeitnehmerfreizügigkeit zu beschränken. Die beschränkenden Wirkungen seien, so der Gerichtshof, nicht zu ungewiss oder zu indirekt, da sie nicht von einem zukünftigen und hypothetischen Ereignis abhingen, sondern von einem Umstand, der zwangsläufig mit der Ausübung des Freizügigkeitsrechts verbunden sei, nämlich von der Wahl des Ortes, an den der Betreffende seinen Wohnsitz verlege. Dass nur marginale Auswirkungen bestehen, weil die Höhe der fraglichen Leistungen und die Anzahl der Betroffenen begrenzt sind, sei irrelevant, da die Grundfreiheiten grundlegende Bestimmungen für die Union darstellten und jede noch so unbedeutende Beeinträchtigung verboten sei.
Jedoch ist zu beachten, dass eine nationale Maßnahme nach der Rechtsprechung des EuGH, auch wenn sie wegen ihrer Ungewissheit und Mittelbarkeit keine Beeinträchtigung der Grundfreiheiten darstellt, keinesfalls unmittelbar aufgrund der Staatsangehörigkeit diskriminieren darf. Dies verbiete das allgemeine Diskriminierungsverbot des Art. 18AEUV, wie der EuGH bereits in den Urteilen Data Delecta Aktiebolag und Saldanha und MTS Securities Corporation entschieden hat ${ }^{67}$. Ein Verstoß gegen Art. 18 AEUV war im Fall Guarnieri deshalb ausgeschlossen, weil es sich im Ausgangsverfahren um einen monegassischen Kläger handelte, der sich nur ausnahmsweise auf den Zollkodex, nicht aber das allgemeine Diskriminierungsverbot stützen konnte 68 .

Unklar in dieser Rechtsprechung bleibt zunächst, warum der EuGH nicht bereits im Rahmen der Warenverkehrsfreiheit die unmittelbare Diskriminierung aus Gründen der Staatsangehörigkeit problematisiert, da auch diese ein Diskriminierungsverbot enthält ${ }^{69}$. Zudem verwundert, dass Art. 18 AEUV angewendet wird, obwohl speziellere Grundfreiheiten, im Fall der Prozesssicherheit regelmäßig die Warenverkehrsfreiheit oder die Dienstleistungsfreiheit, anwendbar und damit im Sinne der Rechtsprechung spezieller sind und die Anwendung des allgemeinen Diskriminierungsverbots ausschließen ${ }^{70}$. Bemerkenswert ist schließlich auch, dass die Eröffnung des sachlichen Anwendungsbereichs des Unionsrechts im Sinne von Art. 18 AEUV damit begründet wird, dass die Prozesskostensicherheit nur für Ausländer »geeignet ist, die wirtschaftliche Betätigung der Wirtschaftsteilnehmer anderer Mitgliedstaaten auf dem Markt des betreffenden Staates zu beeinträchtigen «, auch wenn sie als solche nicht dazu bestimmt ist, eine kaufmännische Tätigkeit zu regeln ${ }^{71}$. De facto wird also doch eine Beeinträchtigung der Warenverkehrs- und Dienstleistungsfreiheit angenommen.

\section{c) Rechtfertigung}

Bei der Rechtfertigung von Beeinträchtigungen gewährt der EuGH den Mitgliedstaaten in einigen Bereichen erhebliche Beurteilungs- und Gestaltungsspielräume. Dies gilt insbesondere für die Bekämpfung des Glücksspiels und die Sicherung des

Art. 34 Rdn. 55; Leible/T. Streinz in: Grabitz/Hilf/Nettesheim (Hrsg), Das Recht der Europäischen Union, 46. Erglfg. 2011 Rdn. 65.

62 So etwa die Interpretation von Generalanwalt Jacobs in seinen Schlussanträgen zur Rs. C-112/00, Slg. 2003, I-5659, Rdn. 65-67 - Schmidberger $\rightarrow$ Schoch JK 11/03, EGV Art. 28/3; vgl hierzu F. C. Mayer EuR 2003, 793 (817).

63 EuGH Urt v 13.3. 1984, Rs 16/83, Slg 1984, 1299, Rdn. 20 - Prantl; vgl auch Urt v 13. 12. 1989, Rs C-49/89, Slg 1989, 4441, Rdn. 8 - Corsica Ferries France; Urt v 1.4.2008, Rs C-212/06, Slg 2008, I-1683, Rdn. 52 - Flämische Pflegeversicherung. Gegen die Auslegung als Spürbarkeitserfordernis Repasi EuZW 2011, 430 (431).

64 EuGH Urt v 7.4.2011, C-291/09, EuZW 2011, 429, Rdn. 15-17 - Francesco Guarnieri \& Cie, mit Anmerkungen von Streinz JuS 2011, $1138 \mathrm{ff}$.; Repasi EuZW 2011, $430 \mathrm{f}$.

65 EuGH Urt v 15.6. 2010, C-211/08, Slg 2010, I-5267, Rdn. 68-73 - Kommission/Spanien.

66 EuGH Urt v 1.4.2008, C-212/06, Slg 2008, I-1683, Rdn. 51-52 - Flämische Pflegeversicherung.

67 EuGH Urt v 26. 9. 1996, C-43/95, Slg 1996 I-4661, Rdn. 16-22 - Data Delecta Aktiebolag; Urt v 2. 10. 1997, C-122/96, Slg 1997, I-5325, Rdn. 25-30 - Saldanha und MTS Securities Corporation.

68 EuGH Urt v 7.4.2011, C-291/09, EuZW 2011, 429, Rdn. 19-20 - Francesco Guarnieri \& Cie.

$69 \mathrm{Vgl}$ schon EuGH Urt v 11. 7. 1974, Slg 1974, 837, Rdn. 7/9-Dassonville, sowie aus der jüngsten Rechtsprechung EuGH Urt v 2.12.2010, C-108/09, EuZW 2011, 112, Rdn. 48 - Ker-Optika.

70 EuGH Urt v 14. 7. 1994, C-379/92, Slg 1994, I-3453, Rdn. 18 - Peralta.

71 EuGH Urt v 26. 9. 1996, C-43/95, Slg 1996 I-4661, Rdn. 10-15 - Data Delecta Aktiebolag. 
Gesundheitssystems (vgl unten II.3.b. und c. sowie II.5.b.). Der Schutz der Vielsprachigkeit in Spanien wurde im Fall UTECA ${ }^{72}$ als ein zwingender Grund des Allgemeininteresses anerkannt. Auch die nationale Identität gemäß Art. 4 II EUV hat der EuGH in seiner neuesten Rechtsprechung zur allgemeinen Freizügigkeit im Rahmen der Rechtfertigung berücksichtigt, teilweise unter dem Gesichtspunkt der öffentlichen Ordnung, teilweise im Rahmen des Schutzes der Landessprache (siehe hierzu die Fälle Sayn-Wittgenstein und Runevic-Vardyn unter I.3.a. und b. ${ }^{73}$. Dies eröffnetauch fürdie Grundfreiheiten neue Rechtfertigungsmöglichkeiten, hat aber auch seine Grenzen: Weiterhin nicht anerkannt wird das Vorbringen mangelnderinnerstaatlicher $\mathrm{Zu}$ ständigkeit wie im Fall der Flämischen Pflegeversicherung ${ }^{74}$. Auch solche Beschränkungen, die sich aus derverfassungsmäßigen Ordnung ergeben (wie zB die föderale Struktur und die Verteilung derGesetzgebungsbefugnisse), können nach Ansicht des EuGH nicht die Nichteinhaltung der aus dem Unionsrecht folgenden Verpflichtungen rechtfertigen.

Generell verlangt der EuGH, dass Beschränkungen der Grundfreiheiten auf systematische und kohärente Weise erfolgen. Nachdem der EuGH diese Kriterien zunächst vor allem bei der Prüfung von mitgliedstaatlichen Regelungen zu den direkten Steuern angewendet hat ${ }^{75}$, werden sie nun allgemein und $\mathrm{zB}$ auch in den Glücksspielfällen und bei der Ausgestaltung sozialer Sicherungssysteme angewendet ${ }^{76}$.

Auch überlässt der EuGH zunehmend die Subsumtion und die Verhältnismäßigkeitsprüfung im Einzelfall den mitgliedstaatlichen Gerichten und gibt nur mehr ${ }^{77}$ oder weniger grobe ${ }^{78}$ Auslegungslinien an die Hand. So überlässt er es etwa im Urteil Kommission/Italien (Kradanhänger) hinsichtlich des Schutzes der Sicherheit des Straßenverkehrs dem Mitgliedstaat zu entscheiden, auf welchem Niveau er diese Sicherheit gewährleisten will und wie dieses Niveau erreicht werden soll und gewährt ihm ausdrücklich einen Beurteilungsspielraum. Die Beweislast des Mitgliedstaates hinsichtlich der Eignung der Maßnahme ginge überdies nicht so weit, dass er »positiv belegen müsste, dass sich das Ziel mit keiner anderen vorstellbaren Maßnahme unter den gleichen Bedingungen erreichen lasse «79. Dies kommt insofern auch dem hinter Art. 267 AEUV stehenden Rechtsgedanken näher, dass der EuGH die Vorgaben zur Auslegung des Unionsrechts vorgibt, während die nationalen Gerichte mit ihrer größeren Sachnähe den konkreten Rechtsstreit entscheiden ${ }^{80}$.

72 EuGH Urt v 5. 3. 2009, C-222/07, Slg 2009, I-1407, Rdn. 27-28-UTECA zu der Pflicht für Fernsehveranstalter zur Vorfinanzierung europäischer Spiel- und Fernsehfilme beizutragen, wobei $60 \%$ dieser Finanzierung Werken vorbehalten war, deren Originalsprache eine der Amtssprachen Spaniens ist.

73 EuGH Urt v 22. 12. 2010, C-208/09, EuGRZ 2011, 25, Rdn. 83, 92 - Sayn-Wittgenstein; Urt v 12.5.2011, C-391/09, noch nicht veröffentlicht, Rdn. 86 Runevic-Vardyn.

74 EuGH Urt v 1. 4. 2008, C-212/06, Slg 2008, I-1683, Rdn. 51-52, 58 - Flämische Pflegeversicherung.

75 EuGH Urt v 28. 1. 1992, C-204/90, Slg 1992, I-249, Rdn. 28 - Bachmann.

76 EuGH Urt v 8. 9. 2010, verb Rs C-316/07 u. a., NVwZ 2010, 1409, Rdn. 88 ff. Stoß u. a. $\rightarrow$ Ehlers JK 1/11, AEUV Art. 49/1; Urt v 10. 3. 2009, C-169/07, Slg 2009, I-1721, Rdn. 55 - Hartlauer; Urt v 21.12. 2011, C-28/09, noch nicht veröffentlicht, Rdn. 126 - Kommission/Österreich (LKW-Fahrverbot).

77 Etwa im Bereich des Glücksspiels EuGH Urt v 15.9. 2011, C-347/09, EuZW 2011, 841, Rdn. 50-51, 57, 65 - Dickinger und Ömer (s. u.); für den Umweltschutz EuGH Urt v 4. 6. 2009, C-142/05, Slg 2009, I-4273, Rdn. 32-43 - Åklagaren/Mickelsson und Roos (siehe hierzu II.2.a.).

78 EuGH Urt v 10. 2. 2009, C-110/05, Slg 2009, I-519, Rdn. 59-69-Kommission/ Italien (Kradanhänger) $\rightarrow$ Schoch JK 1/10, EGV Art. 28/12 (siehe hierzu II.2.a.).

79 Ebd, Rdn. $65 \mathrm{f}$.

80 Fortsetzung in den nächsten beiden JURA Heften. 